\title{
Spin-dependent magnetotransport through a ring due to spin-orbit interaction
}

\author{
B. Molnár and F. M. Peeters \\ Departement Natuurkunde, Universiteit Antwerpen (Campus Drie Eiken), B-2610 Antwerpen, Belgium
}

P. Vasilopoulos

Department of Physics, Concordia University, 1455 de Maisonneuve Quest, Montréal, Quebec, Canada H3G 1 M8

(Received 16 September 2003; revised manuscript received 4 December 2003; published 30 April 2004)

\begin{abstract}
Electron transport through a one-dimensional ring connected with two external leads, in the presence of spin-orbit interaction (SOI) of strength $\alpha$ and a perpendicular magnetic field is studied. Applying Griffith's boundary conditions we derive analytic expressions for the reflection and transmission coefficients of the corresponding one-electron scattering problem. We generalize earlier conductance results by Nitta et al. [Appl. Phys. Lett. 75, 695 (1999)] and investigate the influence of $\alpha$, temperature, and a weak magnetic field on the conductance. Varying $\alpha$ and temperature changes the position of the minima and maxima of the magnetic-field dependent conductance, and it may even convert a maximum into a minimum and vice versa.
\end{abstract}

DOI: 10.1103/PhysRevB.69.155335 PACS number(s): 72.25.-b, 71.70.Ej, 03.65.Vf, 85.35.-p

\section{INTRODUCTION}

Recently, much attention has been paid to the manipulation of the spin degrees of freedom of conduction charges in low-dimensional semiconductor structures. An important feature of the electron transport in multiply connected systems is that the conductance shows signatures of quantum interference that depend on the electromagnetic potentials: Aharonov-Bohm and Aharonov-Casher effect. ${ }^{1-10}$ A comprehensive review of results for metallic rings is given in Ref. 11. Many devices have been proposed to utilize additional topological phases acquired by the electrons traveling through quantum circuits. ${ }^{1,12-15}$ Nitta et al. proposed a spininterference device ${ }^{1}$ allowing considerable modulation of the electric current. This device is a one-dimensional ring connected with two external leads, made of a semiconductor structure in which the Rashba spin-orbit interaction (SOI) (Ref. 16) is the dominant spin-splitting mechanism. The key idea was that, even in the absence of an external magnetic field, the difference in the Aharonov-Casher phase ${ }^{3,6}$ acquired between carriers, traveling clockwise and counterclockwise, would produce interference effects in the spinsensitive electron transport. By tuning the strength $\alpha$ of the SOI the phase difference could be changed, hence the conductance could be modulated. Nitta et al. ${ }^{1}$ found that the conductance $G$ is given approximatively by

$$
G \sim \frac{e^{2}}{h}\left[1+\cos \left(2 \pi \alpha \frac{a m^{*}}{\hbar^{2}}\right)\right],
$$

where $a$ is the radius of the ring and $m^{*}$ the effective mass of the carriers. It is of interest to verify the validity of this strong sinusoidal modulation of the conductance, predicted by Eq. (1).

The Rashba field involved in Ref. 1 results from the asymmetric confinement along the direction $(z)$ perpendicular to the plane of the ring. A similar study but with this field tilted away from the $z$ direction, by an angle $\phi$, was made in Ref. 17. The resulting Rashba field is weaker since the radial part of the confinement is much weaker ${ }^{18}$ but this was not elaborated in Ref. 17. The transmission coefficient of Ref. 17 coincides with ours for $\phi=0$ but it is less general in two important aspects: it is valid only for zero temperature and in the absence of a magnetic field whereas ours is free from these limitations.

In this paper we present an exact, analytic treatment of the influence of the SOI on the electron transport through the spin-interference device of Ref. 1. Applying Griffith's boundary conditions ${ }^{19,20}$ at the junction points we solve the corresponding scattering problem analytically, obtain the correct form of the conductance $G$, and show how for large $\alpha$ it is modulated approximately as predicted by Eq. (1). Further, we assess the influence of a weak magnetic field on this conductance, indicate the spin-filtering properties of the ring, and generalize the result to finite temperatures. These latter aspects were not studied at all in Ref. 17.

The paper is organized as follows. In Sec. II we solve the one-electron problem for a ring in the presence of SOI at zero magnetic field and apply Griffith's boundary conditions. In Sec. III we evaluate in detail the transmission and reflection coefficients and the zero-temperature conductance. In Sec. IV we reevaluate the conductance in the presence of a weak magnetic field and point out the relevance of the results to spin filtering. In Sec. V we present the finite-temperature conductance and some numerical results. Concluding remarks follow in Sec. VI and details about the spin eigenstates and probability currents are given in the Appendix.

\section{ONE-ELECTRON PROBLEM}

\section{A. Hamiltonian}

In the presence of SOI the Hamiltonian operator for a one-dimensional ring structure is given by Ref. 21 ,

$$
\begin{aligned}
\hat{H}= & -\hbar \Omega \frac{\partial^{2}}{\partial \varphi^{2}}-i \hbar \omega_{s o}\left(\cos \varphi \sigma_{x}+\sin \varphi \sigma_{y}\right) \frac{\partial}{\partial \varphi} \\
& -i \frac{\hbar \omega_{s o}}{2}\left(\cos \varphi \sigma_{y}-\sin \varphi \sigma_{x}\right),
\end{aligned}
$$


where $\sigma_{x}, \sigma_{y}$, and $\sigma_{z}$ are the Pauli matrices. The parameter $\Omega$ denotes $\hbar / 2 m^{*} a^{2}$ and $\omega_{s o}=\alpha / \hbar a$ is the frequency associated to the SOI. The Rashba field we consider here results from the asymmetric confinement along the direction $(z)$ perpendicular to the plane of the ring. The parameter $\alpha$ represents the average electric field along the $z$ direction and is assumed to be a tunable quantity. For an InGaAs-based twodimensional electron gas, $\alpha$ can be controlled by a gate voltage with typical values in the range $(0.5-2.0)$ $\times 10^{-11} \mathrm{eVm} .{ }^{22,23}$ Writing the Pauli matrices in cylindrical coordinates,

$$
\sigma_{r}=\cos \varphi \sigma_{x}+\sin \varphi \sigma_{y}, \quad \sigma_{\varphi}=\cos \varphi \sigma_{y}-\sin \varphi \sigma_{x},
$$

and using $\partial \sigma_{r} / \partial \varphi=\sigma_{\varphi}$ one can recast the Hamiltonian in the more compact form,

$$
\hat{H}=\hbar \Omega\left(-i \frac{\partial}{\partial \varphi}+\frac{\omega_{s o}}{2 \Omega} \sigma_{r}\right)^{2} .
$$

An irrelevant additive constant term $\omega_{s o}^{2} / 4 \Omega$ has been neglected in Eq. (2). It should be emphasized that this Hamiltonian is a Hermitian operator, ${ }^{21}$ under proper boundary conditions, in contrast to the non-Hermitian one used in Ref. 1. As can be seen above, the SOI enters Eq. (4) as the spindependent vector potential $\left(\omega_{s o} / 2 \Omega\right) \sigma_{r}$. It is convenient to introduce the dimensionless Hamiltonian

$$
H=\frac{1}{\hbar \Omega} \hat{H}=\left(-i \frac{\partial}{\partial \varphi}+\frac{\omega_{s o}}{2 \Omega} \sigma_{r}\right)^{2} .
$$

Then, as outlined in the Appendix, one can solve the eigenvalue problem in a straightforward manner. The energy spectrum $E_{n}^{(\mu)}$ and unnormalized eigenstates $\Psi_{n}^{(\mu)}$ (the normalization depends on the boundary conditions), labeled by the index $\mu=1,2$, are found to be

$$
\begin{gathered}
E_{n}^{(\mu)}=\left(n-\Phi_{A C}^{(\mu)} / 2 \pi\right)^{2}, \\
\Psi_{n}^{(\mu)}(\varphi)=e^{i n \varphi} \chi_{n}^{(\mu)}(\varphi) .
\end{gathered}
$$

Here the mutually orthogonal spinors $\chi^{(\mu)}(\varphi)$ can be expressed in terms of the eigenvectors $\left(\begin{array}{l}1 \\ 0\end{array}\right),\left(\begin{array}{l}0 \\ 1\end{array}\right)$ of the Pauli matrix $\sigma_{z}$ as

$$
\begin{gathered}
\chi_{n}^{(1)}(\varphi)=\left(\begin{array}{c}
\cos \frac{\theta}{2} \\
e^{i \varphi} \sin \frac{\theta}{2}
\end{array}\right), \\
\chi_{n}^{(2)}(\varphi)=\left(\begin{array}{c}
\sin \frac{\theta}{2} \\
-e^{i \varphi} \cos \frac{\theta}{2}
\end{array}\right),
\end{gathered}
$$

with the angle $\theta$ given by

$$
\theta=2 \arctan \left(\Omega-\sqrt{\Omega^{2}+\omega_{s o}^{2}}\right) / \omega_{s o} .
$$

The spin-dependent term $\Phi_{A C}^{(\mu)}$ is the Aharonov-Casher phase

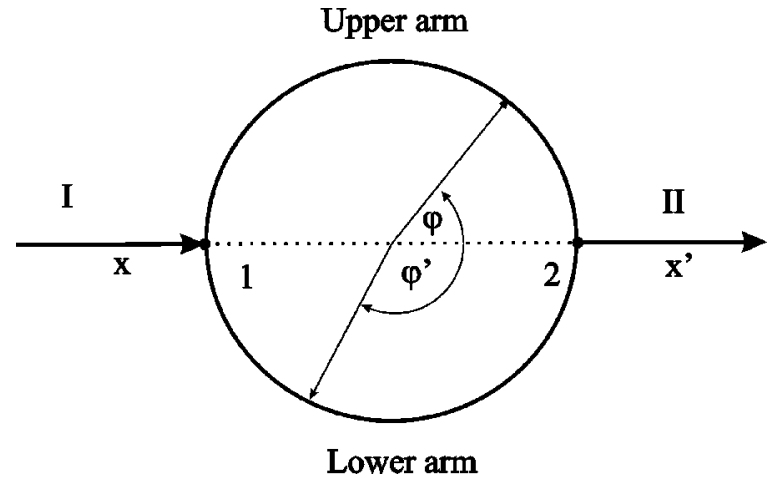

FIG. 1. Device geometry and the local coordinates $\left(x, x^{\prime}, \varphi\right.$, and $\varphi^{\prime}$ ) pertaining to different parts of the ring.

$$
\Phi_{A C}^{(\mu)}=-\pi\left[1+(-1)^{\mu}\left(\omega_{s o}^{2}+\Omega^{2}\right)^{1 / 2} / \Omega\right] .
$$

Until now we have not specified the boundary conditions and solved only the time-independent Schrödinger equation. However, it can be seen from Eqs. (6a) and (6b) that whatever the boundary conditions, in the presence of SOI the solution of the Schrödinger equation differs from the unnormalized, free-energy eigenstates only in the phase factor $\exp \left(i \Phi_{A C}^{(\mu)} / 2 \pi\right)$. In words Eq. (6b) means that the unnormalized spinor $\Psi_{n}^{(\mu)}$ picks up the Aharonov-Casher phase $\Phi_{A C}^{(\mu)}$ upon encircling the ring once.

\section{B. Device geometry and boundary conditions}

The ring connected to two leads is shown in Fig. 1 with the local coordinate systems attached to the different regions of the device. If the ring is not connected to any leads the natural boundary condition is that the wave function has to be single valued when the argument $\varphi$ is increased by an integral multiple of $2 \pi$; this entails that the quantum number $n$ [see Eq. (6b)] must be integer. Connecting the ring to external leads alters this condition. In this case it is appropriate to apply a spin-dependent version of the Griffith's boundary conditions $^{19,20}$ at the intersections as we will specify below. This reduces the electron transport through the spininterference device to an exactly solvable, one-dimensional scattering problem. According to these boundary conditions at each junction: (i) the wave function must be continuous, and (ii) the spin probability current density must be conserved.

In the present problem the total wave function in the incoming and the outgoing lead can be expanded in terms of spinors $\chi^{(\mu)}$ of Eqs. (7a) and (7b) as

$$
\begin{gathered}
\Psi_{I}(x)=\sum_{\mu=1,2} \Psi_{I}^{(\mu)}(x) \chi^{(\mu)}(\pi), \quad x \in[-\infty, 0], \\
\Psi_{I I}\left(x^{\prime}\right)=\sum_{\mu=1,2} \Psi_{I I}^{(\mu)}\left(x^{\prime}\right) \chi^{(\mu)}(0), \quad x^{\prime} \in[0, \infty],
\end{gathered}
$$

respectively. (See Fig. 1 for the local coordinates $x$ and $x^{\prime}$.) The coefficients are the single spin-wave functions $\Psi_{I}^{(\mu)}(x)$ and $\Psi_{I I}^{(\mu)}\left(x^{\prime}\right)$ having the form 


$$
\begin{gathered}
\Psi_{I}^{(\mu)}(x)=\left(e^{i k x} f_{\mu}+e^{-i k x} r_{\mu}\right), \\
\Psi_{I I}^{(\mu)}\left(x^{\prime}\right)=e^{i k x^{\prime}} t_{\mu}
\end{gathered}
$$

respectively, where $k$ denotes the incident wave number, $f_{1}$ $=\cos (\gamma / 2)$ and $f_{2}=\sin (\gamma / 2)$. As it can be seen, $r_{\mu}$ is the reflection coefficient while $t_{\mu}$ is the transmission coefficient for spin polarization $\mu(\mu=1,2)$. In a similar fashion the wave functions corresponding to the upper and lower arms of the ring can be written as

$$
\begin{gathered}
\Psi_{u p}(\varphi)=\sum_{\mu=1,2} \Psi_{u p}^{(\mu)}(\varphi) \chi^{(\mu)}(\varphi), \quad \varphi \in[0, \pi], \quad \\
\Psi_{\text {low }}\left(\varphi^{\prime}\right)=\sum_{\mu=1,2} \Psi_{\text {low }}^{(\mu)}\left(\varphi^{\prime}\right) \chi^{(\mu)}\left(-\varphi^{\prime}\right), \quad \varphi^{\prime} \in[0, \pi],
\end{gathered}
$$

respectively (see Fig. 1 for coordinates). The corresponding wave functions read

$$
\begin{gathered}
\Psi_{u p}^{(\mu)}(\varphi)=\sum_{j=1}^{2} a_{j}^{\mu} e^{i n_{j}^{\mu} \varphi}, \\
\Psi_{\text {low }}^{(\mu)}\left(\varphi^{\prime}\right)=\sum_{j=1}^{2} b_{j}^{\mu} e^{-i n_{j}^{\mu} \varphi^{\prime}} .
\end{gathered}
$$

Here the real numbers $n_{j}^{\mu}(j=1,2)$,

$$
n_{j}^{\mu}=(-1)^{j} k a+\Phi_{A C}^{(\mu)} / 2 \pi,
$$

are the solutions of the equation $k^{2} a^{2}=E_{n^{\mu}}^{\mu}$ ensuring the conservation of energy. The coefficients $r_{\mu}, t_{\mu}, a_{j}^{\mu}$, and $b_{j}^{\mu}$ are not independent: they are connected to each other via Griffith's boundary conditions. First applying the continuity conditions for the wave functions $\Psi_{I I}^{(\mu)}(0)=\Psi_{u p}^{(\mu)}(0)$ $=\Psi_{\text {low }}^{(\mu)}(0)$ and $\Psi_{I}^{(\mu)}(0)=\Psi_{u p}^{(\mu)}(\pi)=\Psi_{\text {low }}^{(\mu)}(\pi)$, one finds

$$
\begin{gathered}
\sum_{j=1}^{2} a_{j}^{\mu}=\sum_{j=1}^{2} b_{j}^{\mu}=t_{\mu}, \\
\sum_{j=1}^{2} a_{j}^{\mu} e^{i n_{j}^{\mu} \pi}=\sum_{j=1}^{2} b_{j}^{\mu} e^{-i n_{j}^{\mu} \pi}=r_{\mu}+f_{\mu} .
\end{gathered}
$$

Now let us turn to the second boundary condition. If one assumes that there are no spin-flip processes at the junctions, one requires that the spin probability currents $J^{\mu}$ for each spin direction $\mu$ should be conserved, i.e.: $J_{u p}^{\mu}+J_{\text {low }}^{\mu}+J_{I(I I)}^{\mu}$ $=0$. As shown in the Appendix, the dimensionless spin currents in the ring arms are found to be

$$
\begin{aligned}
J_{u p}^{\mu}(\varphi)= & 2 \operatorname{Re}\left\{\left(\Psi_{u p}^{(\mu)} \chi^{(\mu)}\right)^{\dagger}\left(-i \partial / \partial \varphi+\omega_{s o} \sigma_{r} / 2 \Omega\right)\right. \\
& \left.\times \Psi_{u p}^{(\mu)} \chi^{(\mu)}\right\} \\
J_{\text {low }}^{\mu}\left(\varphi^{\prime}\right)= & 2 \operatorname{Re}\left\{\left(\Psi_{\text {low }}^{(\mu)} \chi^{(\mu)}\right)^{\dagger}\left(-i \partial / \partial \varphi^{\prime}-\omega_{s o} \sigma_{r}^{\prime} / 2 \Omega\right)\right. \\
& \left.\times \Psi_{\text {low }}^{(\mu)} \chi^{(\mu)}\right\}
\end{aligned}
$$

where $\sigma_{r}^{\prime}\left(\varphi^{\prime}\right)=\sigma_{r}\left(\varphi=-\varphi^{\prime}\right)=\cos \varphi^{\prime} \sigma_{x}-\sin \varphi^{\prime} \sigma_{y}$ because of the orientation of the coordinate system in the lower arm is opposite to that in the upper arm. The currents in the leads are given by

$$
\begin{gathered}
J_{I}^{\mu}(x)=2 a \operatorname{Re}\left\{\left(\Psi_{I}^{(\mu)} \chi^{(\mu)}\right)^{\dagger}(-i \partial / \partial x) \Psi_{I}^{(\mu)} \chi^{(\mu)}\right\}, \\
J_{I I}^{\mu}\left(x^{\prime}\right)=2 a \operatorname{Re}\left\{\left(\Psi_{I I}^{(\mu)} \chi^{(\mu)}\right)^{\dagger}\left(-i \partial / \partial x^{\prime}\right) \Psi_{I I}^{(\mu)} \chi^{(\mu)}\right\} .
\end{gathered}
$$

Here it should be emphasized that the spinors $\chi^{(\mu)}(\mu$ $=1,2$ ) are obviously the eigenstates of the operator $-i \partial / \partial \varphi+\left(\omega_{s o} / 2 \Omega\right) \sigma_{r}$, which commutes with $\hat{H}$ given by Eq. (4). Therefore $J^{\mu}$ are well-defined conserved spincurrent densities in the ring. Using the previous requirement $\Psi_{I(I I)}^{(\mu)}=\Psi_{u p}^{(\mu)}=\Psi_{\text {low }}^{(\mu)}$ at the junctions, the conservation of the spin-current densities can be simply written as

$$
\left.\partial \Psi_{u p}^{(\mu)}\right|_{\varphi=0(\pi)}+\left.\partial \Psi_{\text {low }}^{(\mu)}\right|_{\varphi^{\prime}=0(\pi)}+\left.a \partial \Psi_{I I(I)}^{(\mu)}\right|_{x^{\prime}(x)=0}=0 .
$$

Evaluating the derivatives, one obtains

$$
\begin{gathered}
\sum_{j=1}^{2} a_{j}^{\mu} \frac{n_{j}^{\mu}}{k a}-\sum_{j=1}^{2} b_{j}^{\mu} \frac{n_{j}^{\mu}}{k a}+t_{\mu}=0, \\
\sum_{j=1}^{2} a_{j}^{\mu} e^{i n_{j}^{\mu} \pi} \frac{n_{j}^{\mu}}{k a}-\sum_{j=1}^{2} b_{j}^{\mu} e^{-i n_{j}^{\mu} \pi} \frac{n_{j}^{\mu}}{k a}+f_{\mu}-r_{\mu}=0 .
\end{gathered}
$$

The variables $r_{\mu}, t_{\mu}$ can be eliminated using Eqs. (15a) and (15b). Then the set of Eqs. (19a) and (19b) is replaced by the linear set of algebraic equations for the coefficients $\left\{a_{j}^{\mu}, b_{j}^{\mu}\right\}$ :

$$
\begin{gathered}
\sum_{j=1}^{2} a_{j}^{\mu} \frac{n_{j}^{\mu}+k a}{k a}-\sum_{j=1}^{2} b_{j}^{\mu} \frac{n_{j}^{\mu}}{k a}=0, \\
\sum_{j=1}^{2} a_{j}^{\mu} e^{i n_{j}^{\mu} \pi} \frac{n_{j}^{\mu}-k a}{k a}-\sum_{j=1}^{2} b_{j}^{\mu} e^{-i n_{j}^{\mu} \pi} \frac{n_{j}^{\mu}}{k a}=-2 f_{\mu} .
\end{gathered}
$$

\section{TRANSMISSION AND REFLECTION COEFFICIENTS AND CONDUCTANCE}

The linear Eqs. (15a) and (15b) together with Eqs. (20a) and (20b) for the variables $a_{j}^{\mu}$ and $b_{j}^{\mu}$ can be written in the matrix form,

$$
M^{\mu}\left[\begin{array}{c}
a_{1}^{\mu} \\
a_{2}^{\mu} \\
b_{1}^{\mu} \\
b_{2}^{\mu}
\end{array}\right]=-2\left[\begin{array}{c}
0 \\
0 \\
0 \\
f_{\mu}
\end{array}\right],
$$

with the matrix $M^{\mu}$ depending only on the wave number $k a$ and $n_{j}^{\mu}$ : 


$$
M^{\mu}=\left[\begin{array}{cccc}
1 & 1 & -1 & -1 \\
e^{i n_{1}^{\mu} \pi} & e^{i n_{2}^{\mu} \pi} & -e^{-i n_{1}^{\mu} \pi} & -e^{-i n_{2}^{\mu} \pi} \\
\frac{n_{1}^{\mu}+k a}{k a} & \frac{n_{2}^{\mu}+k a}{k a} & -\frac{n_{1}^{\mu}}{k a} & -\frac{n_{2}^{\mu}}{k a} \\
\frac{n_{1}^{\mu}-k a}{k a} e^{i n_{1}^{\mu} \pi} & \frac{n_{2}^{\mu}-k a}{k a} e^{i n_{2}^{\mu} \pi} & -\frac{n_{1}^{\mu}}{k a} e^{-i n_{1}^{\mu} \pi} & -\frac{n_{2}^{\mu}}{k a} e^{-i n_{2}^{\mu} \pi}
\end{array}\right] .
$$

Now let us calculate the transmission $\left(t_{\mu}\right)$ and reflection $\left(r_{\mu}\right)$ coefficients which are connected to the incoming spinor according to the following equations:

$$
\begin{aligned}
& \left(\begin{array}{l}
t_{1} \\
t_{2}
\end{array}\right)=T\left(\begin{array}{l}
\cos \frac{\gamma}{2} \\
\sin \frac{\gamma}{2}
\end{array}\right)=\left[\begin{array}{cc}
T_{1} & 0 \\
0 & T_{2}
\end{array}\right]\left(\begin{array}{l}
\cos \frac{\gamma}{2} \\
\sin \frac{\gamma}{2}
\end{array}\right), \\
& \left(\begin{array}{l}
r_{1} \\
r_{2}
\end{array}\right)=R\left(\begin{array}{c}
\cos \frac{\gamma}{2} \\
\sin \frac{\gamma}{2}
\end{array}\right)=\left[\begin{array}{cc}
R_{1} & 0 \\
0 & R_{2}
\end{array}\right]\left(\begin{array}{c}
\cos \frac{\gamma}{2} \\
\sin \frac{\gamma}{2}
\end{array}\right) .
\end{aligned}
$$

Both diagonal matrices $T$ and $R$ can be expressed in terms of the inverse of the $4 \times 4$ matrix $M^{\mu}$ in the manner

$$
\begin{gathered}
T_{\mu}=-2\left[\left(M^{\mu}\right)_{1,4}^{-1}+\left(M^{\mu}\right)_{2,4}^{-1}\right], \\
R_{\mu}=-2\left[e^{i n_{1}^{\mu} \pi}\left(M^{\mu}\right)_{1,4}^{-1}+e^{i n_{2}^{\mu} \pi}\left(M^{\mu}\right)_{2,4}^{-1}+1 / 2\right] .
\end{gathered}
$$

Calculating the fourth row of the inverse matrix gives

$$
\begin{gathered}
T_{\mu}=-8 i \cos \left(\Theta_{\mu} \pi\right) \sin \left(\Lambda_{\mu} \pi\right) / d_{\mu}, \\
R_{\mu}=\left[\cos \left(2 \Lambda_{\mu} \pi\right)-1\right] k a / \Lambda_{\mu} d_{\mu}+4\left[\cos \left(2 \Theta_{\mu} \pi\right)\right. \\
\left.-\cos \left(2 \Lambda_{\mu} \pi\right)\right] \Lambda_{\mu} / \mathrm{kad}_{\mu},
\end{gathered}
$$

with the following notations:

$$
\begin{gathered}
d_{\mu}=\left[\cos \left(2 \Lambda_{\mu} \pi\right)-1\right] k a / \Lambda_{\mu}+4\left[\cos \left(2 \Lambda_{\mu} \pi\right)\right. \\
\left.-\cos \left(2 \Theta_{\mu} \pi\right)\right] \Lambda_{\mu} / k a-4 i \sin \left(2 \Lambda_{\mu} \pi\right), \\
\Lambda_{\mu}=\left(n_{2}^{\mu}-n_{1}^{\mu}\right) / 2, \quad \Theta_{\mu}=\left(n_{2}^{\mu}+n_{1}^{\mu}\right) / 2 .
\end{gathered}
$$

One can verify that for each spin polarization $\mu(\mu=1,2)$,

$$
\left|T_{\mu}\right|^{2}+\left|R_{\mu}\right|^{2}=1
$$

Here we would like to point out that the expressions for $T_{\mu}$ and $R_{\mu}$ above are quite general. They are still valid for other Hamiltonians than the one used, provided the spinors $\chi_{n_{1}^{\mu}}^{\mu}$ and $\chi_{n}{ }_{2}^{\mu}$, which travel clockwise and counterclockwise, respectively, are along the same direction.

In the present case $\Lambda_{\mu}=k a$ and $\Theta_{\mu}=\Phi_{A C}^{(\mu)} / 2 \pi$. Consequently the concrete expression for the transmission amplitudes reads

$$
T_{\mu}=\frac{8 i \cos \left(\Phi_{A C}^{(\mu)} / 2\right) \sin (k a \pi)}{1-5 \cos (2 k a \pi)+4 \cos \Phi_{A C}^{(\mu)}+4 i \sin (2 k a \pi)} .
$$

In the Landauer formalism the conductance is given by

$$
G=\frac{e^{2}}{h} \sum_{\mu, \lambda=1}^{2}\left|T_{\mu \lambda}\right|^{2}
$$

In the present case the off-diagonal elements $T_{12}$ and $T_{21}$ of the transmission matrix are zero. Inserting Eq. (28) in Eq. (29) we obtain the exact conductance at zero temperature in the form

$$
G=\left(e^{2} / h\right) g_{0}\left(k, \Delta_{A C}\right)\left[1-\cos \left(\Delta_{A C}\right)\right],
$$

where the dimensionless coefficient $g_{0}$ is

$$
\begin{aligned}
& g_{0}\left(k, \Delta_{A C}\right) \\
& \quad=\frac{64 \sin ^{2}(k a \pi)}{\left[1-5 \cos (2 k a \pi)-4 \cos \left(\Delta_{A C}\right)\right]^{2}+16 \sin ^{2}(2 k a \pi)} .
\end{aligned}
$$

Here $\Delta_{A C}=\left(\Phi_{A C}^{(1)}-\Phi_{A C}^{(2)}\right) / 2=\pi\left[\left(2 m * a / \hbar^{2}\right)^{2} \alpha^{2}+1\right]^{1 / 2}$ is the half of the difference between the phases accumulated by the different spinors. Comparing Eq. (30) with the approximate formula (1) one can see that the conductance oscillates with $\cos \left(\Delta_{A C}\right)$ in a more complex manner. For large values of the Rashba parameter $\alpha$ an essential difference is a $\pi$ phase shift in the oscillation; however, the period remains the same. An important feature is the presence of the factor $\cos \left(\Delta_{A C}\right)$ in the denominator of Eq. (31). This makes $g_{0}$ not a constant equal to 1 , as found in Ref. 1, but a quantity that depends on $\Delta_{A C}$ and the incident energy through $k$. The full dependence of $g_{0}$ on $\Delta_{A C}$ for different temperatures, including $T=0$, is shown in Sec. V.

Figure 2 shows the conductance $G$ versus $\Delta_{A C}$ at different wave numbers $k$. Because $G$ is an even and periodic function of $k a$ (with period 1), it is sufficient to consider only the half period $k a \in[0,1 / 2]$. One can see that $k \approx 0$ (or for $k a \approx l$ $\in \mathbb{N}$ ) the conductance tends to a discontinuous function which is nonzero only at $\Delta_{A C}=\pi+2 n \pi$ ( $n$ is integer) with value $2 e^{2} / h$. This dependence of $G$ on $k a$ is absent in Eq. (1). We note in passing that a transmission coefficient formally equivalent to Eq. (30) was derived earlier in Ref. 17 with very few details and starting with a Hamiltonian in 


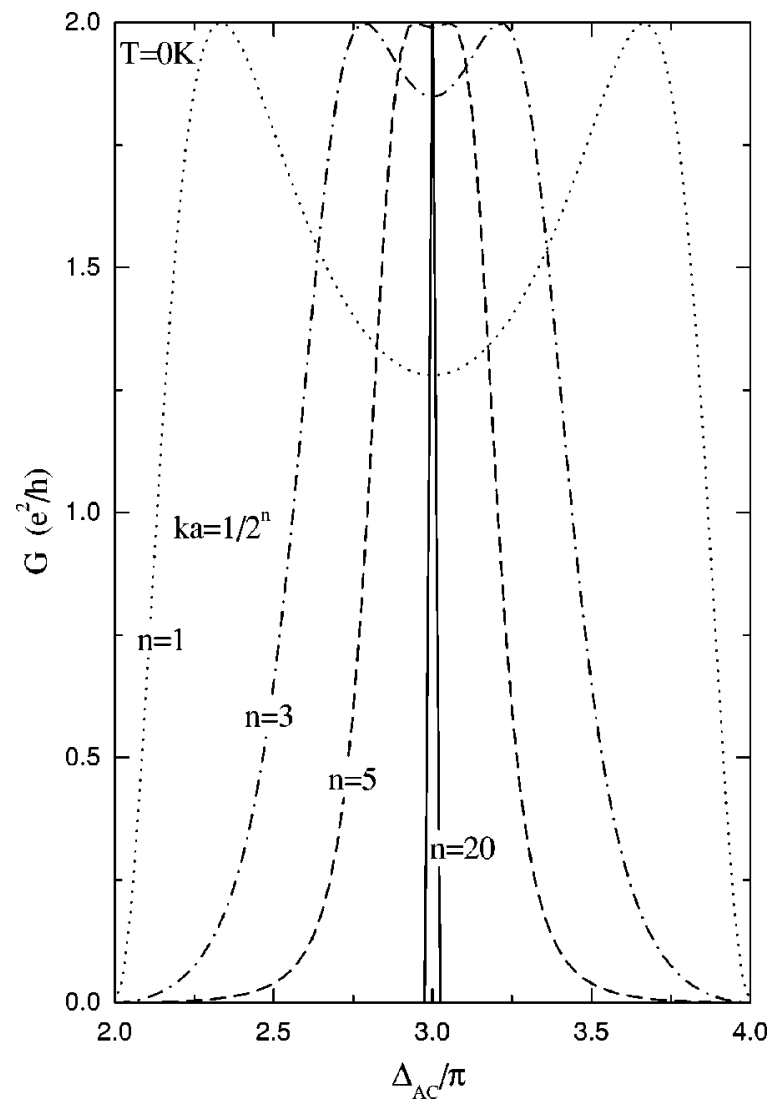

FIG. 2. Dependence of the conductance $G$ on the AharonovCasher phase $\Delta_{A C}$ for different incident wave numbers $k a$ at zero temperature. $G$ is a periodic and even function of $k a$, hence $k a$ was considered only in the interval $[0,1 / 2]$.

which the Rashba field is tilted away from the $z$ direction by an angle $\phi$. It coincides with ours for $\phi=0$. As shown below, however, ours takes into account finite temperatures and a weak magnetic field whereas that of Ref. 17 does not. In addition, we give the reflection coefficient in Eq. (25b).

\section{WEAK MAGNETIC PERTURBATION}

Our analytic result can be easily extended to the case of a weak magnetic perturbation. Let us suppose that an external magnetic field $\vec{B}$ normal to the plane of the ring is present. Then the vector potential can be chosen to be tangential

$$
\vec{A}=(B a / 2) \vec{e}_{\varphi}
$$

First we take the effect of the magnetic flux $\Phi=a \oint \vec{A} d \vec{\varphi}$ encircled by the ring into consideration. It means that we have to change the momentum operator $-i \hbar \nabla$ in the Hamiltonian with $-i \hbar \nabla-e \vec{A}$ ("minimal coupling" substitution). This leads to the appearance of the magnetic flux $\Phi / \Phi_{0}$ in the Hamiltonian, where $\Phi_{0}=h / e$ is the unit of flux, if the Zeeman term $g * \vec{B} \cdot \vec{S}$ is neglected. ${ }^{5}$ Then the AharonovBohm phase picked up by an electron encircling this magnetic flux

$$
\Phi_{A B}=2 \pi \Phi / \Phi_{0}=\pi e B a^{2} / \hbar,
$$

and the dimensionless Hamiltonian in question reads

$$
H=\left(-i \frac{\partial}{\partial \varphi}-\frac{\Phi_{A B}}{2 \pi}+\frac{\omega_{s o}}{2 \Omega} \sigma_{r}\right)^{2} .
$$

When the Zeeman term is present, the interaction between the electron spin and a relatively weak magnetic field $B$ can be treated by perturbation theory. Using the dimensionless field strength $b=g^{*} e B / 4 m \Omega$ the perturbation of the Hamiltonian (34) is

$$
H_{p}=b \sigma_{z}=(g * m * / m) \Phi_{A B} \sigma_{z},
$$

where $m$ is the bare electron mass and $g *$ the effective gyromagnetic ratio. The matrix elements of $H_{p}$ in the basis of the normalized eigenstates $\left|\Psi_{n}^{\mu}\right\rangle=\Psi_{n}^{(\mu)}(\varphi) / \sqrt{2 \pi}$ are obtained as

$$
\begin{gathered}
\left\langle\Psi_{n}^{\mu}\left|H_{p}\right| \Psi_{n}^{\mu}\right\rangle= \\
=(-1)^{\mu+1}\left(g^{*} m^{*} / m\right) \Phi_{A B} \cos \vartheta \\
\left\langle\Psi_{n}^{1}\left|H_{p}\right| \Psi_{n}^{2}\right\rangle=\left(g^{*+1} C_{\vartheta} * / m\right) \Phi_{A B} \sin \vartheta .
\end{gathered}
$$

In the first-order approximation one neglects the off-diagonal elements; this is reasonable if they are small, i.e., if $\left(g^{*} m^{*} / m\right) \Phi_{A B} \ll k^{2} a^{2}$. To first order the eigenspinors are not perturbed and their direction is still specified by the angle $\vartheta$ given by Eq. (8). Using the identity

$$
\cos \vartheta=\frac{1-\tan ^{2}(\vartheta / 2)}{1+\tan ^{2}(\vartheta / 2)}=\pi / \Delta_{A C},
$$

we obtain the energies, including the first-order corrections,

$$
E_{n}^{\mu}=\left(n-\frac{\Phi_{A B}}{2 \pi}-\frac{\Phi_{A C}^{(\mu)}}{2 \pi}\right)^{2}-(-1)^{\mu} \frac{g^{*} m^{*} \pi}{m \Delta_{A C}} \Phi_{A B} .
$$

The equation of energy conservation $k^{2} a^{2}=E_{n^{\mu}}^{\mu}$ has the solutions

$$
\begin{gathered}
n_{1}^{\mu}=-\sqrt{k^{2} a^{2}+(-1)^{\mu} C_{\vartheta}}+\Phi_{A B} / 2 \pi+\Phi_{A C}^{(\mu)} / 2 \pi, \\
n_{2}^{\mu}=\sqrt{k^{2} a^{2}+(-1)^{\mu} C_{\vartheta}}+\Phi_{A B} / 2 \pi+\Phi_{A C}^{(\mu)} / 2 \pi .
\end{gathered}
$$

Because the eigenspinors are not modified within this approximation the transmission matrix elements are given again by Eq. (25a) but with the parameters $\Lambda_{\mu}$ and $\Theta_{\mu}$ replaced, respectively, by

$$
\Lambda_{\mu}=\left(n_{2}^{\mu}-n_{1}^{\mu}\right) / 2=\sqrt{k^{2} a^{2}+(-1)^{\mu} C_{\vartheta}},
$$

and

$$
\Theta_{\mu}=\left(n_{2}^{\mu}+n_{1}^{\mu}\right) / 2=\Phi_{A B} / 2 \pi+\Phi_{A C}^{(\mu)} / 2 \pi=\Phi / \Phi_{0}+\Phi_{A C}^{(\mu)} / 2 \pi .
$$

This leads to the transmission coefficient 


$$
T_{\mu}=\frac{8 i \cos \left(\Phi_{A B} / 2+\Phi_{A C}^{(\mu)} / 2\right) \sin \left(C_{\mu} k a \pi\right)}{C_{\mu}^{-1}-\left(C_{\mu}^{-1}+4\right) \cos \left(2 C_{\mu} k a \pi\right)+4 \cos \left(\Phi_{A B}+\Phi_{A C}^{(\mu)}\right)+4 i \sin \left(2 C_{\mu} k a \pi\right)},
$$

where $C_{\mu}=\sqrt{1+(-1)^{\mu} C_{\vartheta} / k^{2} a^{2}}$. The resulting magnetoconductance reads

$$
G=\frac{e^{2}}{h}\left[\left|T_{1}\right|^{2}+\left|T_{2}\right|^{2}\right] .
$$

At this point one can envisage an application of the device as a spin filter. Assuming one can tune the phases $\Phi_{A B}$ and $\Phi_{A C}^{(\mu)}$ (via the magnetic field and the Rashba strength $\alpha$ ) independently, one can make the ring almost transparent with high transmission probability only for electrons with spin quantum number $\mu=1$ (2) and totally opaque with $\mu=2$ (1). For instance, if one sets $\Phi_{A B}+\Phi_{A C}^{(1)}$ and $\Phi_{A B}+\Phi_{A C}^{(2)}$ to be $(2 p+1) \pi$ and $2 q \pi$ into Eq. (41), where $q$ and $p$ are integers, respectively, one obtains

$$
\begin{gathered}
\left|T_{1}\right|^{2}=0 \\
\left|T_{2}\right|^{2}=\left(1+\frac{1+8 C_{2}}{32\left(C_{2}\right)^{2}}\left[1-\cos \left(2 C_{2} k a \pi\right)\right]\right)^{-1} .
\end{gathered}
$$

As can be seen, $\left|T_{2}\right|^{2}$ has maxima equal to 1 and minima equal to $\left(1+1 / 4 C_{2}\right)^{-2}$ at integer and half-integer values of
$C_{2} k a$, respectively. Due to the inequality $\left(g^{*} m^{*} / m\right) \Phi_{A B}$ $\ll k^{2} a^{2}$ we have $C_{2} \sim 1$; hence the efficiency of the filtering process is higher than $64 \%$.

\section{TEMPERATURE DEPENDENCE OF THE CONDUCTANCE}

\section{A. Explicit expression}

The conductance at finite temperatures is given by

$$
G(T)=-\frac{e^{2}}{h} \sum_{\mu=1,2} \int_{0}^{\infty} d E \frac{\partial f(E, \mu, T)}{\partial E}\left|T_{\mu}(E)\right|^{2},
$$

where $f(E, \mu, T)$ is the Fermi function, $T$ is the temperature, and $T_{\mu}(E)$ is the single spin-transmission coefficient. In the absence of magnetic field the conductance can be written as

$$
G=\left(e^{2} / h\right) g_{T}\left(k_{F}, \Delta_{A C}\right)\left(1-\cos \Delta_{A C}\right),
$$

where the explicit form of the temperature depending coefficient $g_{T}\left(k_{F}, \Delta_{A C}\right)$ is given by

$$
g_{T}\left(k_{F}, \Delta_{A C}\right)=\int_{0}^{\infty} d \zeta \zeta \frac{\left(32 T_{F} / T\right) \cosh ^{-2}\left[\left(\zeta^{2}-\bar{\mu}\right) T_{F} / 2 T\right] \sin ^{2}\left(\zeta k_{F} a \pi\right)}{\left[1-5 \cos \left(2 \zeta k_{F} a \pi\right)-4 \cos \left(\Delta_{A C}\right)\right]^{2}+16 \sin ^{2}\left(2 \zeta k_{F} a \pi\right)} .
$$

Here $\bar{\mu}$ is the (dimensionless) chemical potential in units of the Fermi energy $E_{F}$ and $T_{F}$ denotes the Fermi temperature. At $T=0$ the derivative of the Fermi function becomes a $\delta$ function, the integration in Eq. (46) can be carried out, and one obtains the previous result $g_{0}$ [Eq. (31)].

In the present of a weak magnetic field $\left(C_{\mu} \approx 1\right)$ the magnetoconductance reads

$$
\begin{aligned}
G= & \frac{e^{2}}{2 h} \sum_{\mu=1,2} g_{T}\left[k_{F}, \Phi_{A B}+(-1)^{\mu} \Delta_{A C}\right] \\
& \times\left\{1-\cos \left[\Phi_{A B}+(-1)^{\mu} \Delta_{A C}\right]\right\} .
\end{aligned}
$$

As can be seen, the total magnetoconductance for weak fields is the sum of the two single spin magnetoconductances having the same functional form $\left(e^{2} / 2 h\right) g_{T}\left(k_{F}, \phi\right)[1-\cos \phi]$ but due to the presence of the SOI they are shifted by the spin-depending phase $\pm \Delta_{A C}$ according to Eq. (47).

\section{B. Numerical results}

To stress the difference between our result and the one of Ref. 1 we plot in Figs. 3(a) and 3(b) the coefficient $g_{0}$ for different values of $k a$ as indicated. As shown, the coefficient $g_{0}\left(\Delta_{A C}\right)$ varies in a rather large range, $0-16$, depending on the value of $k a$. The largest deviations from 1 occur at the end of the period $\Delta_{A C} / \pi=2$ and 4. Agreement with Ref. 1 is obtained only for values $\Delta_{A C} / \pi$ in the neighborhood of 3 . This range is the widest (approximately between 2.5 and 3.5) for $k a$ half integer. For $k a$ integer this range collapses into one single point because with this wave number the coefficient $g_{0}$ is discontinuous having the value 1 only at $\Delta_{A C} / \pi=3$ and otherwise zero.

In Figs. 4(a)-4(d) we investigate the temperature dependence of the amplitude $g_{T}$ of the oscillations for different values of wave number $k_{F} a=20,20.25$, and 20.5. The temperature is expressed in units of the Fermi temperature $T_{F}$. As seen, for $k_{F} a$ half integer raising the temperature reduces the value of $g_{T}$; however, for values $k_{F} a$ closer to an integer the coefficient $g_{T}$ increases until its peaks reach a value around 4. This happens for temperature $T \approx 0.05 T_{F}$; and as one can see, by then the dependence on the fractional part of $k_{F} a$ has already been washed out, too. For a ring of radius 


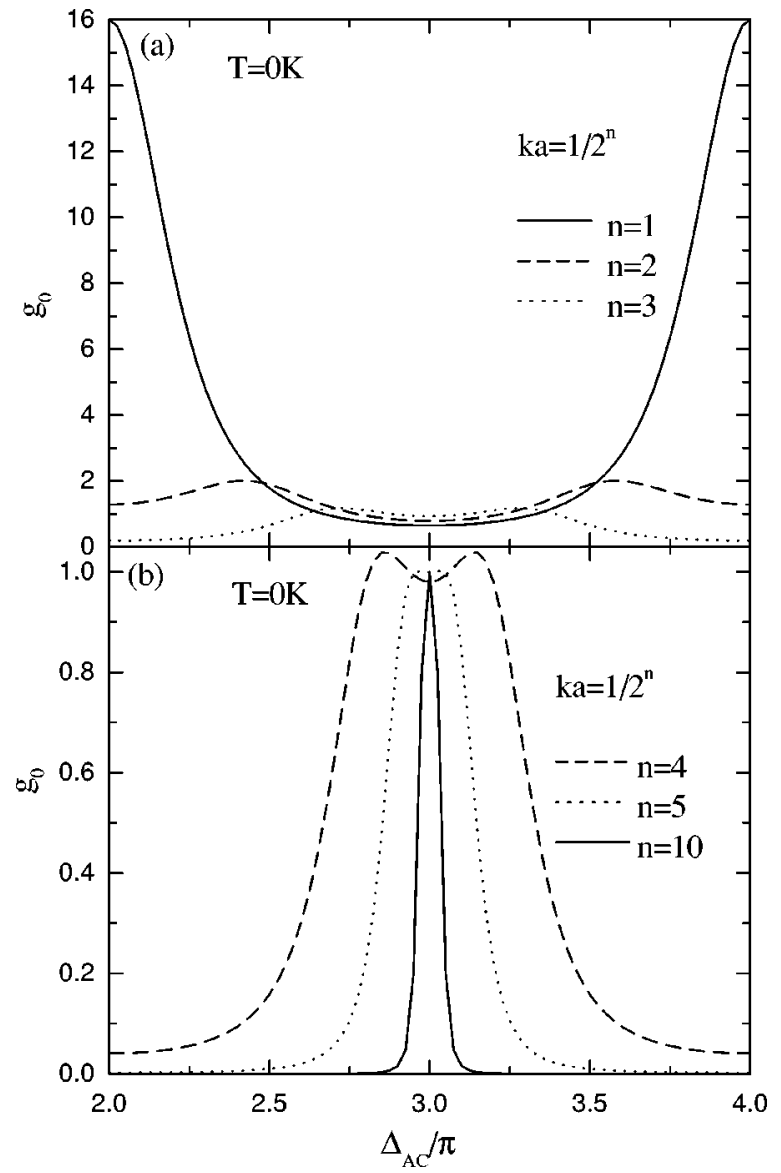

FIG. 3. Dependence of the zero-temperature coefficent $g_{0}$ on the Aharonov-Casher phase $\Delta_{A C}$ for different wave numbers $k a$.

$a=0.25 \mu \mathrm{m}$ and a Fermi wave number $k_{F}=20.5 / a$, with the effective mass of InAs: $m^{*}=0.023$, the Fermi energy $E_{F}$ and the Fermi temperature $T_{F}$ are $11.13 \mathrm{meV}$ and $129.27 \mathrm{~K}$, respectively. With this choice of parameters $T=0.05 T_{F}$ above corresponds to $6.46 \mathrm{~K}$. Further increasing the temperature, now by larger steps as shown in Fig. 4(d), we find that $g_{T}$ decreases more slowly. We also notice that the curves in Fig. 4(d) for different $k_{F} a$ and these high temperatures practically collapse onto a single curve since for small differences in $k_{F} a, \Delta k_{F} / k_{F} \approx 0.025$, the difference in $g_{T}$ is always less than $10^{-5}$ and results from this high-temperature behavior of the integrand in Eq. (46).

For the sake of completeness, in Figs. 5(a), 5(b), and 5(c) we present the conductance $G=\left(e^{2} / h\right) g_{T}\left[1-\cos \left(\Delta_{A C}\right)\right]$ for the same temperatures and values of $k_{F} a$ as in Fig. 4. One can see that by increasing the temperature the "camel hump-like" pattern for $k_{F} a$ around half integers disappears and $G$ becomes less sensitive to the fractional part of $k_{F} a$. A more complete dependence of the conductance on $k a$ and $\alpha$ at zero temperature is shown in Fig. 6. As can be inferred, e.g., by moving along lines of constant $\alpha$ or $k a$, the conductance depends in a complex manner on $\alpha$ and $k a$. Note that the dependence of the conductance on $k a$ is completely absent in Eq. (1).

Figures 7(a) and 7(b) show the oscillations of the magnetoconductance versus magnetic field $B$ in units of $B_{0}$

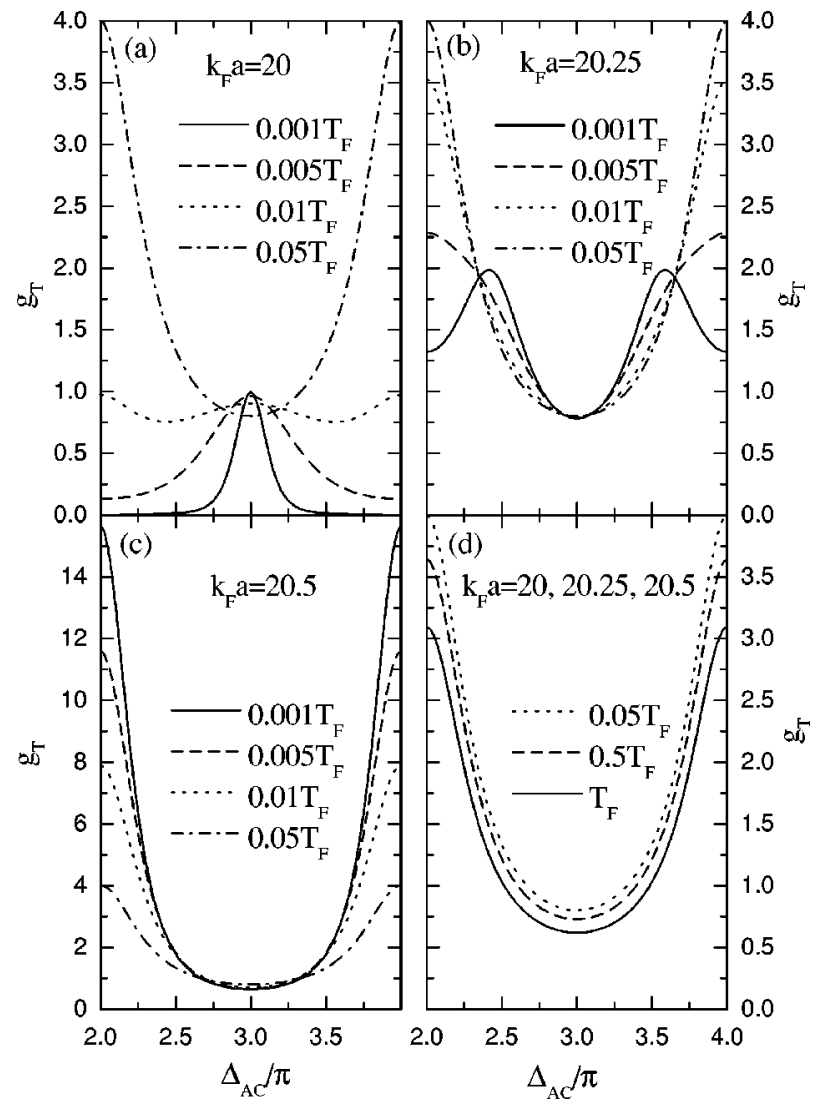

FIG. 4. Dependence of the coefficent $g_{T}$ on the AharonovCasher phase $\Delta_{A C}$ for different temperatures $T$ and different values of the Fermi wave number $k_{F} a$.

$=\Phi_{0} /\left(a^{2} \pi\right)=21.06 \mathrm{mT}$ for various values of the SOI strength $\alpha$ and for fixed Fermi wave number $k_{F} a=20.5$ at $T=0.001 T_{F}$ and $T=0.05 T_{F}$, respectively. In both figures the values of $\alpha$ were chosen such that with the above parameters $m^{*}$ and $a$ they correspond to an Aharonov-Casher phase shift $\Delta_{A C}$ equal to $5 \pi / 4,3 \pi / 2$, and $2 \pi$ for $\alpha=0.497 \alpha_{0}$, $0.741 \alpha_{0}$, and $1.148 \alpha_{0}$, respectively, with $\alpha_{0}=10^{-11} \mathrm{eV} \mathrm{m}$. One can see that the presence of SOI can alter the period of the oscillations, which in its absence is equal to $B_{0}{ }^{11}$

In order to get better insight into the positions of extrema in the magnetoconductance we plotted in Figs. 8(a) and 8(b) those positions as function of the SOI strength $\alpha$ for fixed temperatures (a) $T=0.001 T_{F}$ and (b) $T=0.05 T_{F}$, respectively. Comparing the figure at large temperature with the one at low temperature, it can be seen that the additional substructure of two maxima and a minimum, which is present at $T=0.001 T_{F}$ and connected with the "camel hump" pattern of the magnetoconductance oscillation, has been contracted into a single maximum. Further, at both temperatures, near certain values of $\alpha$, minima (maxima) disappear, and instead of them, a new maximum (minimum) appears, in other words a bifurcation occurs, in the oscillation of the magnetoconductance at $B=0, B_{0} / 2$, and $B_{0}$. These intersections of maximum and minimum curves correspond to saddle points on the surface of the conductance $G$ depending on both $B$ and $\alpha$. To show more clearly how changing the strength $\alpha$ can convert a minimum (maximum) to a 


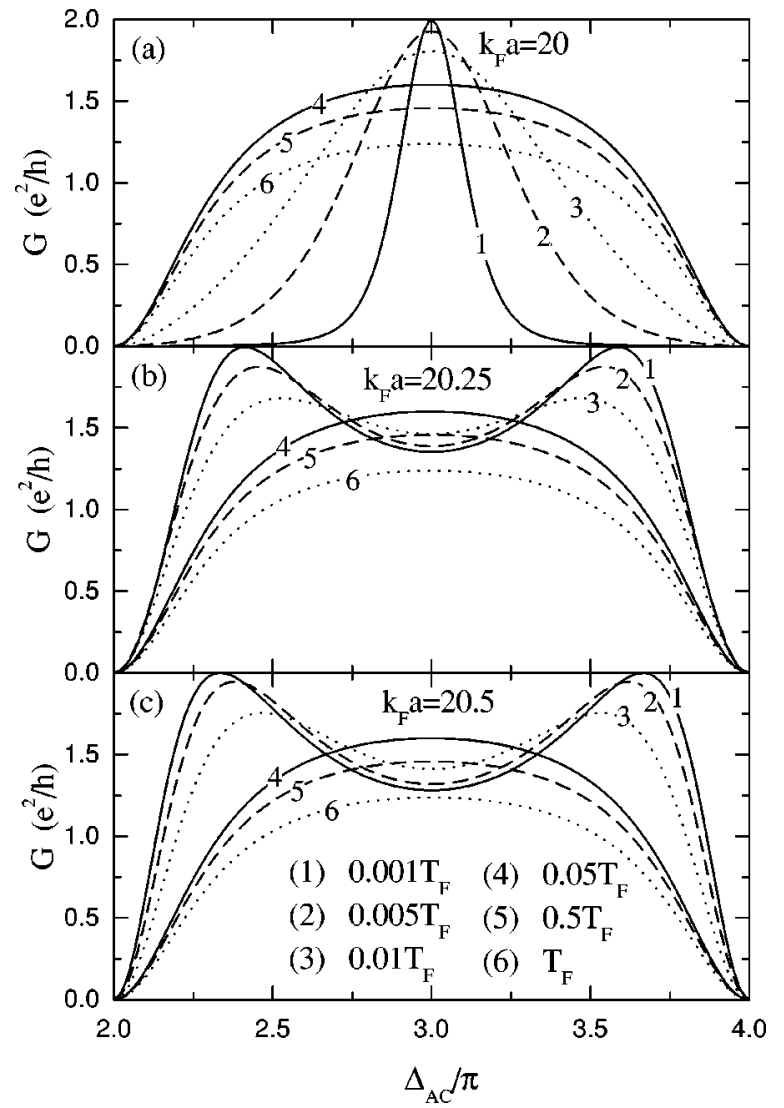

FIG. 5. Dependence of the conductance $G$ on the AharonovCasher phase $\Delta_{A C}$ at different temperatures $T$ and Fermi wave numbers $k_{F} a$.

maximum (minimum), we plot in Figs. 9(a) and 9(b) the magnetoconductance in the neighborhood of two saddle points for temperatures $T=0.001 T_{F}$ and $T=0.05 T_{F}$, respectively. For instance, in Fig. 9(b) one can see that for a rela-

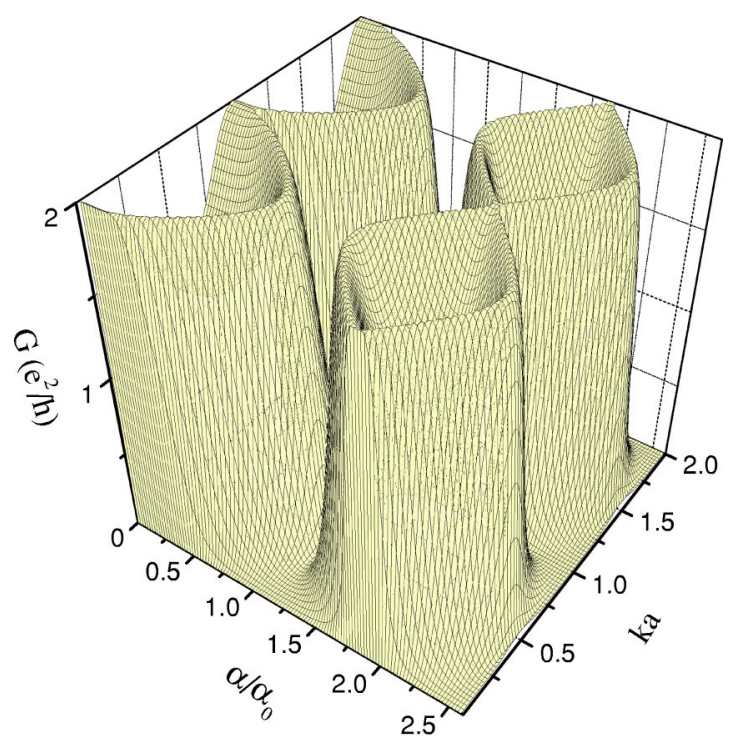

FIG. 6. Dependence of $G$ on the SOI strength $\alpha$ and $k a$ at zero temperature; $\alpha_{0}$ is the value $10^{-11} \mathrm{eV} \mathrm{m}$.

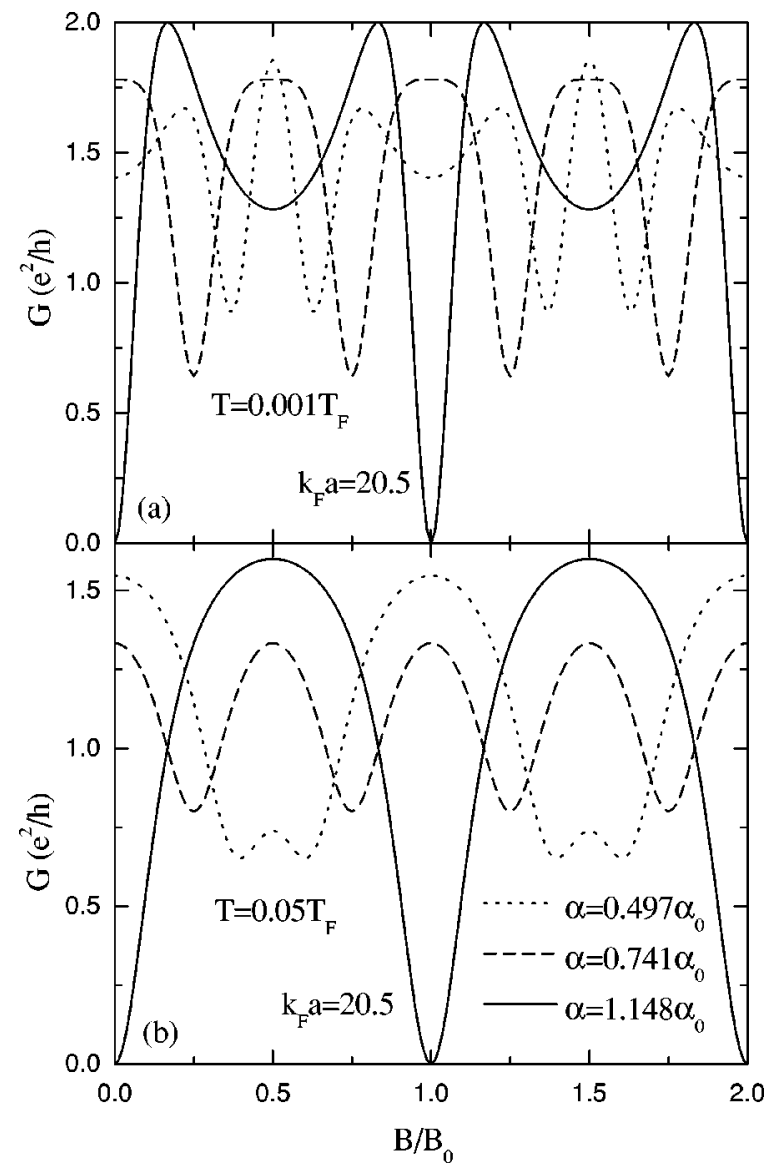

FIG. 7. Magnetoconductance for various values of $\alpha$, in units of $\alpha_{0}=10^{-11} \mathrm{eV} \mathrm{m}$, and at low (a) and high (b) temperatures.

tively small increase (decrease) in $\alpha$ around $0.40 \alpha_{0}\left(1.02 \alpha_{0}\right)$ a minimum turns into a maximum surrounded with two minima at $B=B_{0} / 2\left(B=B_{0}\right)$.

\section{CONCLUDING REMARKS}

We derived an exact expression for the zero-temperature conductance of a one-dimensional ring connected to two leads in the presence of SOI. In addition, we generalized the result to finite temperatures and weak magnetic fields for which the Zeeman term can be treated by perturbation theory. Since we used the Landauer-Büttiker formalism, the conductance expressions are valid in the ballistic regime.

As specified in the text, the zero-temperature conductance is not as simple as presented in Ref. 1. Apart from the phase shift $\pi$ between the two expressions cf., Eqs. (1) and (30), the quantity $g_{0}$ is not equal to 1 , as deduced from Eq. (1), but depends on the strength $\alpha$ of the SOI, on the incident energy, and the temperature, cf. Sec. V. We attribute this difference to the non-Hermitian Hamiltonian and also to the boundary conditions used in Ref. 1. However, the sinusoidal dependence of $G$ on $\alpha$ as predicted in Ref. 1 is recovered by our exact expression only in the limit of large values of $\alpha$.

The results presented here are valid for a strictly onedimensional ring. They can be extended to rings of finite width $w$ provided the inequality $w \ll a$ holds and an infinite- 


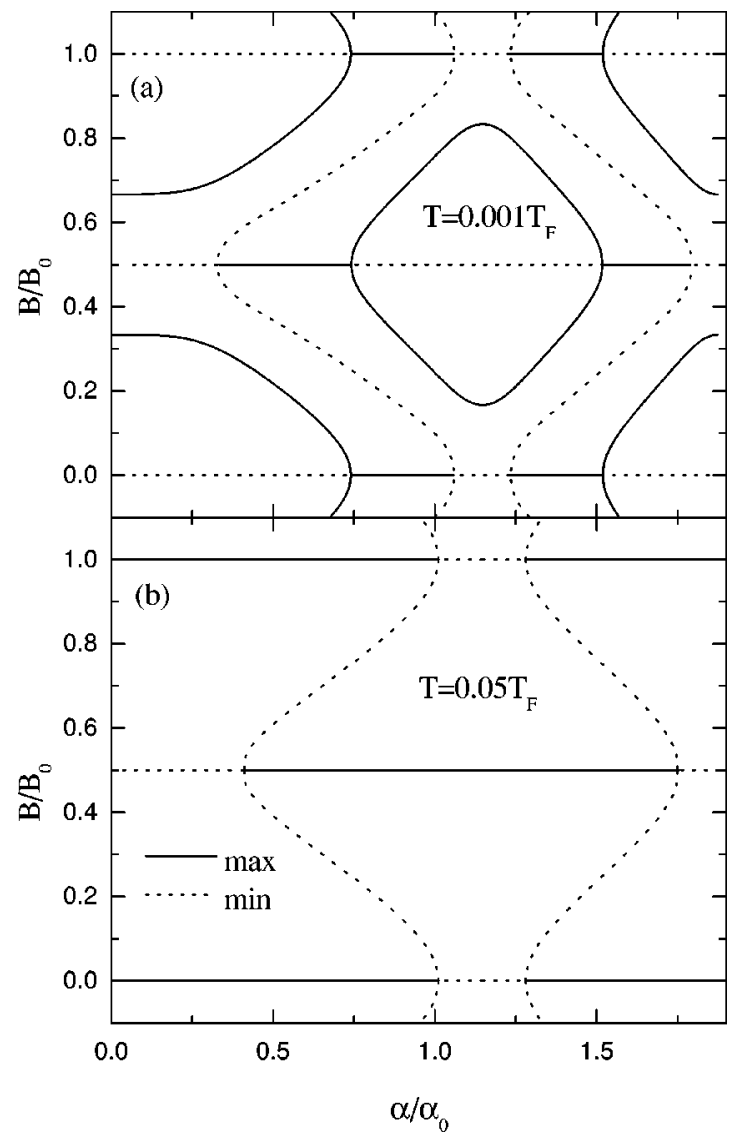

FIG. 8. Positions of extrema in the magnetoconductance oscillation as a function of $\alpha$ at low (a) and high (b) temperature.

wall confinement is assumed along the radial direction. In this case the radial and angular motion are decoupled and the energy levels are shifted by $\hbar^{2} l^{2} / 2 m * w^{2}$, where $l$ is an integer. The results presented in our paper correspond then to the lowest $l=1$ mode.

\section{ACKNOWLEDGMENTS}

This work was supported by the Belgian Interuniversity Attraction Poles (IUAP), the Flemish Concerted Action (GOA) Program, the Flemish Science Foundation (FWO$\mathrm{Vl}$, the EU-CERION program, the Flemish-Hungarian Bilateral Program, and by the Canadian NSERC Grant No. OGP0121756. One of us (B.M.) was financially supported by DWTC to promote $\mathrm{S} \& \mathrm{~T}$ collaboration between Central and Eastern Europe.

\section{APPENDIX}

Below we give some details of the derivation of the unnormalized eigenstates $\Psi_{n}^{\mu}$, Eq. (6b), and of the spin probability currents in Eqs. (16a) and (16b).

(i) Eigenfunctions $\Psi_{n}^{(\mu)}(\varphi)$ : It is sufficient to solve the eigenvalue problem $H \Psi(\varphi)=E \Psi(\varphi)$,

$$
\left(-i \partial / \partial \varphi+\omega_{s o} \sigma_{r} / 2 \Omega\right) \Psi(\varphi)=\Lambda \Psi(\varphi),
$$

with energy eigenvalue $E=\Lambda^{2}$. Writing $\Psi(\varphi)$ in the form

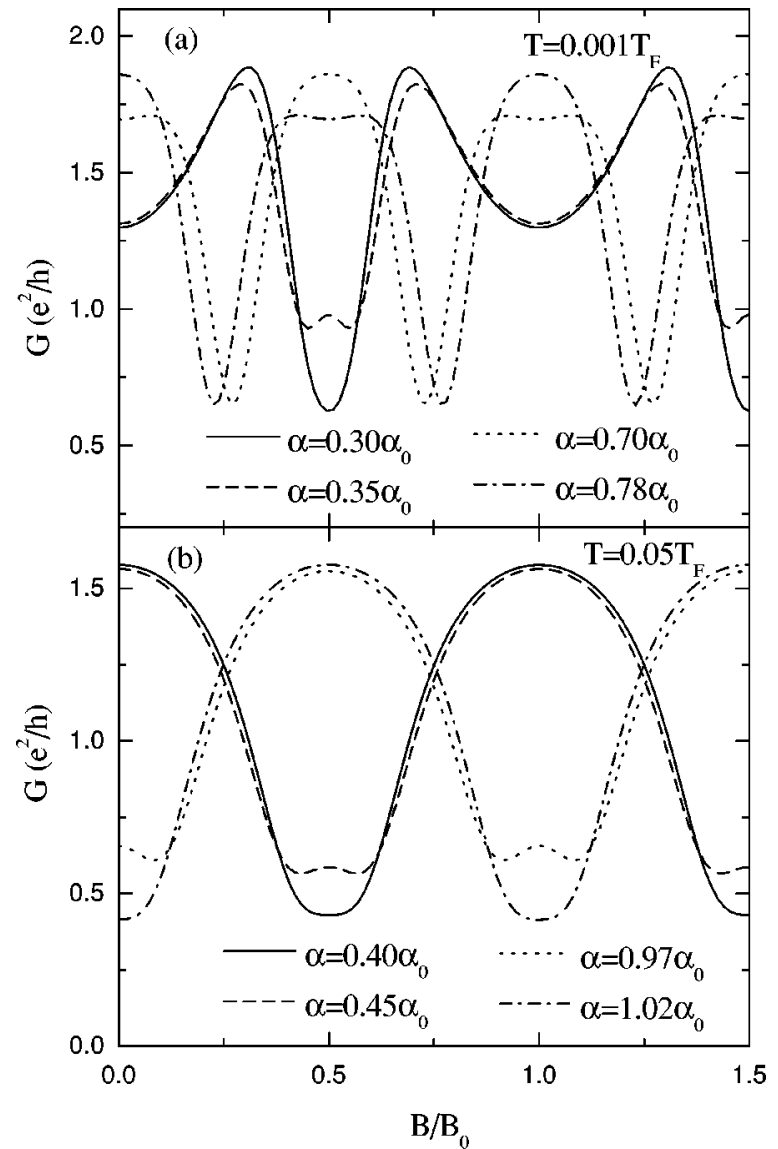

FIG. 9. Minimum-maximum conversions in the oscillations of the magnetoconductance due to changes in the SOI strength $\alpha$ for two values of the temperature.

$$
\Psi(\varphi)=e^{i n \varphi} \chi(\varphi)=e^{i n \varphi}\left(\begin{array}{c}
a \\
b e^{i \varphi}
\end{array}\right)
$$

we obtain

$$
\left(-i \partial / \partial \varphi+\omega_{s o} \sigma_{r} / 2 \Omega\right) \chi(\varphi)=(\Lambda-n) \chi(\varphi) .
$$

Using $\sigma_{r}=\left(\begin{array}{ll}0 & e^{-i \varphi} \\ e^{+i \varphi} & 0\end{array}\right)$ (in the basis $\left(\begin{array}{l}1 \\ 0\end{array}\right),\left(\begin{array}{l}0 \\ 1\end{array}\right)$ of the eigenstates of the Pauli matrix $\sigma_{z}$ ) we obtain

$$
\left(\begin{array}{cc}
0 & \omega_{s o} / 2 \Omega \\
\omega_{s o} / 2 \Omega & 1
\end{array}\right)\left(\begin{array}{l}
a \\
b
\end{array}\right)=(\Lambda-n)\left(\begin{array}{l}
a \\
b
\end{array}\right) .
$$

The eigenvalues of the latter equation are $1 / 2$ $+(-1)^{\mu} \sqrt{1 / 4+\omega_{s o}^{2} / 4 \Omega^{2}}=-\Phi_{A C}^{(\mu)} / 2 \pi$, where $\mu=1,2$. The coefficients of the corresponding eigenvectors can be chosen as $\quad a_{1}=\cos \theta / 2, \quad b_{1}=\sin \theta / 2, \quad a_{2}=\sin \theta / 2, \quad$ and $\quad b_{2}$ $=-\cos \theta / 2, \quad$ with $\quad \tan \theta / 2=\left[1 / 2-\sqrt{1 / 4+\omega_{s o}^{2} / 4 \Omega^{2}}\right]$ $\times\left(2 \Omega / \omega_{\text {so }}\right)=\left[\Omega-\sqrt{\Omega^{2}+\omega_{\text {so }}^{2}}\right] / \omega_{\text {so }}$. The resulting energy eigenvalues and unnormalized eigenfunctions are given, respectively, by Eqs. (6a) and (6b).

(ii) Spin probability currents: The derivation is given as follows.

(a) We denote a two-component spinor by $\Psi=\left(\begin{array}{l}\Psi_{1} \\ \Psi_{2}\end{array}\right)$ and its complex conjugate by $\bar{\Psi}$. Further, we introduce the bilin- 
ear product by $(\Phi, \Psi)=\Phi_{1} \Psi_{1}+\Phi_{2} \Psi_{2}$. Notice that this $i s$ not a scalar product of the Hilbert space. One can show that the following continuity equation is valid for the spinor $\Psi$ obeying the Schrödinger equation $i \partial \Psi / \partial t=H \Psi$ with $H$ given by Eq. (5):

$$
\frac{\partial \rho}{\partial t}+\frac{\partial J}{\partial \varphi}=0
$$

where the probability density is $\rho=(\bar{\Psi}, \Psi)$ and the probability current density $J=2 \operatorname{Re}\{(\bar{\Psi},(-i \partial \Psi / \partial \varphi$ $\left.\left.+\left(\omega_{s o} / 2 \Omega\right) \sigma_{r} \Psi\right)\right\}$.

Proof. We start with the Schrödinger equation $i \partial \Psi / \partial t$ $=H \Psi$ written explicitly as

$$
i \frac{\partial \Psi}{\partial t}=-\frac{\partial^{2} \Psi}{\partial^{2} \varphi}-i \frac{\omega_{s o}}{\Omega} \sigma_{r} \frac{\partial \Psi}{\partial \varphi}-i \frac{\omega_{s o}}{2 \Omega} \frac{\partial \sigma_{r}}{\partial \varphi} \Psi+\frac{\omega_{s o}^{2}}{4 \Omega^{2}} \Psi,
$$

take its and complex conjugate, and consider the products $(\Psi, H \Psi)$ and $(\overline{H \Psi}, \Psi)$,

$$
\begin{aligned}
(\Psi, H \Psi)= & -\left(\Psi, \frac{\partial^{2} \Psi}{\partial^{2} \varphi}\right)-i \frac{\omega_{s o}}{\Omega}\left(\bar{\Psi}, \sigma_{r} \frac{\partial \Psi}{\partial \varphi}\right) \\
& -i \frac{\omega_{s o}}{2 \Omega}\left(\bar{\Psi}, \frac{\partial \sigma_{r}}{\partial \varphi} \Psi\right)+\frac{\omega_{s o}^{2}}{4 \Omega^{2}}(\bar{\Psi}, \Psi), \\
(\overline{H \Psi}, \Psi)= & -\left(\frac{\partial^{2} \Psi}{\partial^{2} \varphi}, \Psi\right)+i \frac{\omega_{s o}}{\Omega}\left(\bar{\sigma}_{r} \frac{\partial \Psi}{\partial \varphi}, \Psi\right) \\
& +i \frac{\omega_{s o}}{2 \Omega}\left(\frac{\partial \bar{\sigma}_{r}}{\partial \varphi} \Psi, \Psi\right)+\frac{\omega_{s o}^{2}}{4 \Omega^{2}}(\Psi, \Psi) .
\end{aligned}
$$

Using the fact $\left(\overline{\sigma_{r} \Phi}, \Psi\right)=\left(\bar{\Phi}, \sigma_{r} \Psi\right)$ the latter product can be written as

$$
\begin{aligned}
(\overline{H \Psi}, \Psi)= & -\left(\frac{\partial^{2} \bar{\Psi}}{\partial^{2} \varphi}, \Psi\right)+i \frac{\omega_{s o}}{\Omega}\left(\frac{\partial \bar{\Psi}}{\partial \varphi}, \sigma_{r} \Psi\right) \\
& +i \frac{\omega_{s o}}{2 \Omega}\left(\bar{\Psi}, \frac{\partial \sigma_{r}}{\partial \varphi} \Psi\right)+\frac{\omega_{\text {so }}^{2}}{4 \Omega^{2}}(\bar{\Psi}, \Psi)
\end{aligned}
$$

The derivative $\partial \rho / \partial t$ is given by $(\partial \bar{\Psi} / \partial t, \Psi)+(\bar{\Psi}, \partial \Psi / \partial t)$ $=i\{(\overline{H \Psi}, \Psi)-(\bar{\Psi}, H \Psi)\}$. Therefore $\partial \rho / \partial t$ can be written as

$$
\begin{aligned}
\frac{\partial \rho}{\partial t}= & i\left\{\left(\Psi, \frac{\partial^{2} \Psi}{\partial^{2} \varphi}\right)-\left(\frac{\partial^{2} \bar{\Psi}}{\partial^{2} \varphi}, \Psi\right)+i \frac{\omega_{s o}}{\Omega}\left(\bar{\Psi}, \sigma_{r} \frac{\partial \Psi}{\partial \varphi}\right)\right. \\
& \left.+i \frac{\omega_{s o}}{\Omega}\left(\frac{\partial \bar{\Psi}}{\partial \varphi}, \sigma_{r} \Psi\right)+i \frac{\omega_{s o}}{\Omega}\left(\bar{\Psi}, \frac{\partial \sigma_{r}}{\partial \varphi} \Psi\right)\right\} .
\end{aligned}
$$

The resulting continuity equation takes the form

$$
\frac{\partial \rho}{\partial t}=i\left\{\frac{\partial\left(\bar{\Psi}, \frac{\partial \Psi}{\partial \varphi}\right)-\left(\frac{\partial \bar{\Psi}}{\partial \varphi}, \Psi\right)}{\partial \varphi}+i \frac{\omega_{s o}}{\Omega} \frac{\partial\left(\bar{\Psi}, \sigma_{r} \Psi\right)}{\partial \varphi}\right\}
$$

and the current $J$ is given by

$$
J=\left\{\left(\overline{-i \frac{\partial \Psi}{\partial \varphi}+\frac{\omega_{s o}}{2 \Omega} \sigma_{r} \Psi, \Psi}\right)+\left(\Psi,-i \frac{\partial \Psi}{\partial \varphi}+\frac{\omega_{s o}}{2 \Omega} \sigma_{r} \Psi\right)\right\}
$$

or

$$
\begin{aligned}
J & =2 \operatorname{Re}\left\{\left(\Psi,-i \frac{\partial \Psi}{\partial \varphi}+\frac{\omega_{s o}}{2 \Omega} \sigma_{r} \Psi\right)\right\} \\
& =2 \operatorname{Re}\left\{\Psi^{\dagger}\left(-i \frac{\partial \Psi}{\partial \varphi}+\frac{\omega_{s o}}{2 \Omega} \sigma_{r} \Psi\right)\right\} .
\end{aligned}
$$

(b) Because the orientation of the coordinate system in the upper arm is opposite to that in the lower arm, the current in the latter is given by $J_{\text {low }}^{\mu}\left(\varphi^{\prime}\right)=-J_{u p}^{\mu}\left(\varphi=-\varphi^{\prime}\right)$. The resulting forms of the two currents are given, respectively, by Eqs. (16a) and (16b).
${ }^{1}$ J. Nitta, F.E. Meijer, and H. Takayanagi, Appl. Phys. Lett. 75, 695 (1999)

${ }^{2}$ Y. Aharonov and D. Bohm, Phys. Rev. 115, 485 (1959).

${ }^{3}$ Y. Aharonov and A. Casher, Phys. Rev. Lett. 53, 319 (1984).

${ }^{4} \mathrm{~J}$. Hamilton, Aharonov-Bohm and other Cyclic Phenomena (Springer, Berlin, 1997).

${ }^{5}$ Y. Meir, Y. Gefen, and O. Entin-Wohlman, Phys. Rev. Lett. 63, 798 (1989).

${ }^{6}$ T.Z. Qian and Z.B. Su, Phys. Rev. Lett. 72, 2311 (1994).

${ }^{7}$ S. Oh and C.M. Ryu, Phys. Rev. B 51, 13441 (1995).

${ }^{8}$ Y.S. Yi, T.Z. Qian, and Z.B. Su, Phys. Rev. B 55, 10631 (1997).
${ }^{9}$ S.L. Zhu and Z.D. Wang, Phys. Rev. Lett. 85, 1076 (2000).

${ }^{10}$ A.G. Aronov and Y.B. Lyanda-Geller, Phys. Rev. Lett. 70, 343 (1993).

${ }^{11}$ R.A. Webb and S. Washburn, Rep. Prog. Phys. 55, 1311 (1992).

${ }^{12}$ J.C. Egues, G. Burkard, and D. Loss, Appl. Phys. Lett. 82, 2658 (2003).

${ }^{13}$ D. Frustaglia, M. Hentschel, and K. Richter, Phys. Rev. Lett. 87, 256602 (2001).

${ }^{14}$ R. Ionicioiu and I. D'Amico, Phys. Rev. B 67, 041307(R) (2003).

${ }^{15}$ J.B. Yau, E.P. De Poortere, and M. Shayegan, Phys. Rev. Lett. 88, 146801 (2003). 
${ }^{16}$ E.I. Rashba, Fiz. Tverd. Tela (Leningrad) 2, 1224 (1960) [Sov. Phys. Solid State 2, 1109 (1960)].

${ }^{17}$ T. Choi, S.Y. Cho, C.M. Ryu, and C.K. Kim, Phys. Rev. B 56, 4825 (1997).

${ }^{18}$ J. Schliemann, J.C. Egues, and D. Loss, Phys. Rev. Lett. 90, 146801 (2003).

${ }^{19}$ S. Griffith, Trans. Faraday Soc. 49, 345 (1953); 49, 4825 (1997).
${ }^{20}$ J.B. Xia, Phys. Rev. B 45, 3593 (1992).

${ }^{21}$ F.E. Meijer, A.F. Morpurgo, and T.M. Klapwijk, Phys. Rev. B 66, 033107 (2002).

${ }^{22}$ J. Nitta, T. Akazaki, H. Takayanagi, and T. Enoki, Phys. Rev. Lett. 78, 1335 (1997).

${ }^{23}$ D. Grundler, Phys. Rev. Lett. 84, 6074 (2000). 